and year) general population. Relative Excess Risks of death were estimated using a multivariable generalised linear model with a Poisson distribution.

Results Overall, 10.782 patients were included; $50.4 \%$ was 65 years or older. Surgery was performed in $30 \%$ of the patients and decreased with increasing age $(\mathrm{p}<0.001)$. Over time, less patients received surgery $(p<0.001)$. Relative survival was increasing in patients that received surgery, adjusted for potential confounders the Relative Excess Risks was 0.7 (95\% CI 0.6 to 0.9 ; $\mathrm{p}<0.001)$ for 65-74 years old patients, 0.5 (95\% CI 0.5 to 0.6 ); $\mathrm{p}<0.001)$ for patients aged $75-84$ and 0.4 (95\% CI 0.3 to $0.6 ; \mathrm{p}<0.001)$ for the patients 85 and older. There were no differences in the independent prognostic factors associated with relative survival (age, grade, tumour size, lymph node involvement, type of metastases, additional treatment).

Conclusion This large retrospective study showed an improved relative survival for all elderly that received local surgery for metastatic breast cancer. However, large prospective randomised trials, including the elderly, are needed to confirm this association.

\section{5-6.3 ADVANCED PATERNAL AGE AND RISK OF DEATH BEFORE THE AGE OF 5 YEARS: A REGISTER-BASED COHORT STUDY}

doi:10.1136/jech.2011.142976b.66

A M Nybo Andersen,* L N Jespersen, M Nissen, L H Mortensen. University of Copenhagen, Copenhagen, Denmark

Introduction An association between paternal age and children's health was suggested by Penrose as early as 1955. More recently, relationships have been suggested between paternal age and specific diseases and fetal death. The association is mainly put down to the increased mutation rate in male germ cells. The aim of this study was to investigate the relationship between paternal age and under 5 -year mortality.

Methods Based on data from Danish population-covering registers, we investigated the relationship between paternal age and under 5 year mortality, including cause-specific mortality, taking maternal age, parity and parental educational levels into account. A total of 1140689 live born children were included in the study. Cox regression models were used to estimate HRs for death during the first 5 years of life.

Results Compared with children born to fathers aged 30-34 years an excess risk was found for children born to fathers aged $45+$ years (HR 1.22; 95\% CI 1.05 to 1.42 ). When only $1-5$ years olds were included the RR rose to $1.70 ; 95 \%$ CI 1.23 to 2.34 . The excess risk for children of fathers aged 45 years or more was primarily attributed to an elevated risk of dying from congenital malformations and malignancies.

Conclusion Children with a father aged 45 years or more have an increased risk of dying before the age of 5 years. The findings are compatible with the hypothesis suggesting increased frequency of point mutations in the fertilising sperm cells from men of advanced age.

\section{5-6.4 LIFECOURSE PREDICTORS OF PHYSICAL ACTIVITY AT AGE 50 YEARS: THE NEWCASTLE THOUSAND FAMILIES STUDY}

doi:10.1136/jech.2011.142976b.67

${ }^{1} \mathrm{~K}$ Mann, ${ }^{* 1} \mathrm{~L}$ Hayes, ${ }^{2} \mathrm{~L}$ Parker, ${ }^{1} \mathrm{M}$ Pearce. ${ }^{1}$ Newcastle University, Newcastle upon Tyne, UK; ${ }^{2}$ Dalhousie University, Halifax, Nova Scotia, Canada

Introduction Beneficial effects of physical activity (PA) on health are well known, but limited evidence exists for lifecourse factors that may influence physical activity levels in adulthood. Our aim was to investigate the relative contributions of a range of factors from across life to variations in PA levels in the Newcastle 1000 Families Study.

Methods Detailed information was collected prospectively during childhood. At age 50 years, 574 study members returned selfcompletion questionnaires. Responses included details of PA levels, collated into four indicators - work, commuting (walking, cycling), household (house work, DIY, gardening) and sport activities. Each was defined by three categories (inactive, less active, most active) and analysed by ordered logistic regression, factor analysis and path analysis.

Results Males had higher levels of work activity $(p=0.010)$ and lower levels of household activity $(p<0.001)$. Increased sport $(p=0.009)$ and household $(p=0.002)$ activity were associated with reduced BMI. Increased sport activity was associated with more advantaged social class $(p=0.004)$ and an increase in work activity with middle classes $(p<0.001)$. Current smoking was associated with reduced sport $(p<0.001)$ and work $(p=0.012)$ activities and higher achieved education with decreased household and commuting activities. Factor analysis identified two components: "sport" and "all other PA", to be considered separately in path analysis which showed highest relative contributors were BMI for other PA and current social class and smoking for sport.

Conclusion The association between PA and BMI should be considered bidirectional. Early life factors were negligible when contemporary factors were addressed with the exception of achieved education.

\section{5-6.5 LIFECOURSE MEASURES OF BODY COMPOSITION AND THE RISK OF KIDNEY CANCER: A SYSTEMATIC REVIEW AND META-ANALYSIS OF OBSERVATIONAL STUDIES}

doi:10.1136/jech.2011.142976b.68

${ }^{1} \mathrm{~J}$ Savovic, ${ }^{*} \mathrm{C}$ Bain, ${ }^{3} \mathrm{R}$ Harris, ${ }^{1} \mathrm{R}$ Beynon, ${ }^{1} \mathrm{~L}$ Zuccolo, ${ }^{1} \mathrm{~J}$ Sterne, ${ }^{4} \mathrm{~S}$ Thomas. ${ }^{1}$ University of Bristol, Bristol, UK; ${ }^{2}$ University of Queensland, Brisbane, Australia; ${ }^{3}$ Health Protection Agency, Colindale, London, UK; ${ }^{4}$ University Hospitals Bristol, Bristol, UK

Introduction Increased body mass index (BMI) is a risk factor for kidney cancer. However, previous reviews on this topic included only studies that reported on mid-life BMI. We carried out an updated and more comprehensive review to describe the association between lifecourse measures of body composition and kidney cancer risk.

Methods We searched MEDLINE, EMBASE, ISI and four other databases in July 2010. We assessed identified studies against prespecified criteria, and extracted data using a standard form. We used fixed and random-effects meta-analyses to derive a pooled $\mathrm{OR}$ and CIs for the association between kidney cancer risk and measures of body composition.

Results We identified 17898 hits; 741 papers were retrieved and assessed. Seventy-three papers met inclusion criteria and will be included in updated meta-analyses. Based on results from our previous search (April 2007, 52 studies), higher BMI was associated with kidney cancer (OR 1.39; 95\% CI 1.30 to 1.47 per $5 \mathrm{~kg} / \mathrm{m}^{2}$ increase), with a linear dose-response observed for most studies. Measures of body composition, including waist circumference, waist-to-hip ratio and weight-cycling showed a similar trend, although derived from a smaller number of studies. We are currently updating our meta-analyses with recent studies, focusing on measurements of body composition other than BMI.

Conclusion The linear dose-response, across a range of BMI, suggests that even mildly overweight individuals may decrease their risk of kidney cancer via small reductions in BMI. Updating evidence on 
other measurements of body composition will further elucidate the relationship between body size and kidney cancer.

\section{5-6.6 INFERTILITY AND MEDITERRANEAN DIETARY PATTERN: A NESTED CASE-CONTROL STUDY}

doi:10.1136/jech.2011.142976b.69

E Toledo,* C Lopez-del Burgo, T Errasti-Alcalá, M Bes-Rastrollo, J J Beunza, M Á Martínez-González, J de Irala. University of Navarra, Pamplona, Navarra, Spain

Introduction Infertility affects approximately 15\% of couples during their reproductive lifetime. We aimed to investigate associations between different dietary patterns (DP) and difficulty for getting pregnant in the SUN Project.

Methods Using data from the SUN (Seguimiento Universidad de Navarra) dynamic prospective cohort of university graduates, we conducted a nested case-control analysis of 485 cases and 1670 controls aged $20-45$ years. Cases were female participants who referred having consulted a doctor due to difficulties for getting pregnant. Controls were female participants not having consulted for this reason and having at least one child. Cases and controls were matched according to age. We performed principal component analyses with orthogonal varimax rotation to determine the main DPs in our cohort. We divided our sample according to quartiles of the empirically-identified DPs and conducted conditional logistic regression models.

Results Out of 9811 women enrolled with $20-45$ years of age, 485 referred having consulted a doctor due to difficulty to getting pregnant. Two main DPs were identified: a Western DP and a Mediterranean-type DP. Once potential confounders were adjusted for, no significant association was observed for a higher adherence to the Western DP (OR 1.09 (95\% CI 0.75 to 1.57)). After adjusting for potential confounders, women with a higher adherence to the Mediterranean-type DP had a lower risk for consulting for difficulty to getting pregnant (OR 0.62 (95\%: 0.39 to 0.99 )).
Conclusion Our data suggest that a higher adherence to the Mediterranean-type DP might have a protective effect on infertility.

\section{Wednesday 10 August 2011 \\ Parallel session 6 \\ 6.1 THE GLOBAL STATUS OF EPIDEMIOLOGY}

\section{Chair: Prof Cesar Victora, Brazil 06-1.1 The GLOBAL STATUS OF EPIDEMIOLOGY}

doi:10.1136/jech.2011.142976b.70

\begin{abstract}
${ }^{1}$ A Mandil, ${ }^{*}$ E Franco, ${ }^{*} \mathrm{~S}$ Barreto, ${ }^{*}$ D Prabhakaran, ${ }^{*} \mathrm{~T}$ Blakely, ${ }^{*}{ }^{6} \mathrm{~J}$ Nachega. ${ }^{*}$ ${ }^{1}$ King Saud University, Saudi Arabia; ${ }^{2}$ Mc Gill University, Canada; ${ }^{3}$ Universidade Federal de Minas Gerais, Brazil; ${ }^{4}$ Centre for Chronic Disease Control, India; ${ }^{5}$ University of Otago, New Zealand; ${ }^{6}$ Stellenbosch University, South Africa
\end{abstract}

To take stock of the current status of epidemiology throughout the world, the International Epidemiological Association commissioned a series of eight reviews that are being published in the International Journal of Epidemiology, starting in February 2011. Each review addresses one region of the world, and starts by describing the burden of disease and the current status of epidemiology, covering human resources, training, academic and research institutions, publication patterns, epidemiologic surveillance institutions, and epidemiologic societies. The reviewers then outline the main future challenges for the development of epidemiologic capacity in their regions. The session will include short presentations by the lead writers from the Western Pacific, Middle-East and North Africa, Latin American and Caribbean, North American, South Asia, SubSaharan Africa regions, followed by a general discussion on the similarities and differences among regions. 